\title{
Identification of potential genes and pathways for response prediction of neoadjuvant chemoradiotherapy in patients with rectal cancer by systemic biological analysis
}

\author{
QILIANG PENG ${ }^{1-3 *}$, KAISU LIN ${ }^{4 *}$, YI SHEN $^{5 *}$, PING ZHOU $^{1-3}$, SHAONAN FAN ${ }^{1-3}$, \\ YUNTIAN SHEN ${ }^{1-3}$ and YAQUN ZHU ${ }^{1-3}$ \\ ${ }^{1}$ Department of Radiotherapy and Oncology, The Second Affiliated Hospital of Soochow University; \\ ${ }^{2}$ Institute of Radiotherapy and Oncology, Soochow University; ${ }^{3}$ Suzhou Key Laboratory for Radiation Oncology, \\ Suzhou, Jiangsu 215004; ${ }^{4}$ Department of Oncology, Nantong Rich Hospital, Nantong, Jiangsu 226010; \\ ${ }^{5}$ Department of Oncology, The Second Affiliated Hospital of Soochow University, Suzhou, Jiangsu 215004, P.R. China
}

Received March 15, 2018; Accepted September 13, 2018

DOI: $10.3892 / 01.2018 .9598$

\begin{abstract}
Currently, neoadjuvant chemoradiotherapy (CRT) followed by radical surgery is the standard of care for locally advanced rectal cancer. However, to the best of our knowledge, there are no effective biomarkers for predicting patients who may benefit from neoadjuvant treatment. The aim of the current study was to screen potential crucial genes and pathways associated with the response to CRT in rectal cancer, and provide valid biological information to assist further investigation of CRT optimization. In the current study, differentially expressed (DE) genes were identified from the tumor samples of responders and non-responders to neoadjuvant CRT in the GSE35452 gene expression profile. Seven hub genes and one significant module were identified from the protein-protein interaction (PPI) network. Functional enrichment analysis of all the DE genes and the hub genes, retrieved from PPI network analysis, revealed their associations with CRT response. Genes were identified that may be used to discriminate patients who would or would not clinically benefit from neoadjuvant CRT. Several important pathways enriched by the DE genes, hub genes and selected module were identified, and revealed to be closely associated with radiation response, including excision repair, homologous recombination, Ras signaling pathway, the forkhead box $\mathrm{O}$ signaling pathway, focal adhesion and the Wnt signaling pathway. In conclusion, the current study demonstrated that the identified gene signatures and pathways
\end{abstract}

Correspondence to: Dr Yaqun Zhu, Department of Radiotherapy and Oncology, The Second Affiliated Hospital of Soochow University, 1055 San Xiang Road, Suzhou, Jiangsu 215004, P.R. China

E-mail: szzhuyaqun@sina.com

*Contributed equally

Key words: rectal cancer, differentially expressed genes, pathways, enrichment analysis may be used as molecular biomarkers for predicting CRT response. Furthermore, combinations of these biomarkers may be helpful for optimizing CRT treatment and promoting understanding of the molecular basis of response differences; this needs to be confirmed by further experiments.

\section{Introduction}

Rectal cancer is one of the most commonly diagnosed malignancies and is one of the leading causes of cancer-associated cases of mortality (1). For patients with locally advanced rectal cancer, neoadjuvant chemoradiotherapy (CRT) followed by radical surgery has been the standard treatment for many years (2). However, the response to CRT varies substantially among patients (3). A number of studies have revealed that pathological complete response is associated with improved clinical outcome $(4,5)$. Biomarkers for early response prediction prior to treatment could be helpful for identifying rectal cancer patients who would not benefit from CRT. This may save potentially nonresponsive patients from unnecessary treatment and possible side effects, and allow alternative treatments, including surgery and adjuvant therapy, to be administered without delay.

A number of studies have revealed a wide variety of genetic and molecular biomarkers that have the potential to predict the response to CRT, including epidermal growth factor receptor, thymidylate synthase and Bax (5-7). However, no previously identified biomarker has changed the approach to CRT treatment or been used to perform clinical trials, due to inadequate sensitivity and specificity. Improved predictive methods are urgently required to discriminate patients who would or would not clinically benefit from neoadjuvant CRT.

Recent technological advances in the genomics of gene expression by DNA microarray have facilitated the simultaneous analysis of a large numbers of genes; this supports the identification of molecular markers with the potential to predict response to CRT at an early time point (8). With the progress of technology, issues have emerged due to differentially expressed (DE) gene lists for the same tumor, identified 
from different microarray studies, demonstrating very low reproducibility, which places a serious impact on further research (9). Considering the heterogeneity and complexity of carcinogenesis, microarray-based biomarker discovery should be established by network paradigms regarding gene interaction, which may eliminate data inconsistencies.

The current study applied an integrative approach to predict novel mRNAs that may be applied to predict chemosensitivity and identify patients with rectal cancer as responders or non-responders to neoadjuvant CRT. Integration of mRNA expression data, mRNA-mRNA interaction data and other types of genomic information may facilitate the identification of mRNA biomarker panels for precision medicine in rectal cancer.

\section{Materials and methods}

Data collection. The gene expression dataset GSE35452 (10) was downloaded from the Gene Expression Omnibus (GEO) database (http://www.ncbi.nlm.nih.gov/geo/). This dataset contained 46 patients with rectal cancer who had been approved to receive preoperative CRT. Biopsy specimens of rectal tumor were collected during colonoscopic examination prior to preoperative CRT, snap-frozen in liquid nitrogen and stored at $-80^{\circ} \mathrm{C}$ until use. Total RNA was isolated from frozen samples and gene expression profiles were determined using Affymetrix Human Genome U133 Plus 2.0 arrays (Thermo Fisher Scientific, Inc., Waltham, MA, USA). The study protocol was approved by the Ethics Committee of Teikyo University (Tokyo, Japan). The current study was also approved by the Ethics Committee of the Second Affiliated Hospital of Soochow University (Suzhou, China).

Response to CRT. The semi-quantitative classification system defined by the Japanese Society for Cancer of the Colon and Rectum was applied to evaluate the response to CRT by histopathological examination of surgically resected specimens (11). Based on the system, no tumor cell necrosis or degeneration was defined as grade 0 , tumor cell necrosis or degeneration in less than two-thirds of the entire lesion was defined as grade 1, prominent tumor cell necrosis or degeneration in more than two-thirds of the entire lesion with viable tumor cells remaining was defined as grade 2, and the presence of no viable tumor cells was defined as grade 3. Tumors were classified as 'responders' (histopathologic regression grade 2 or 3) and 'nonresponders' (histopathologic regression grade 0 or 1 ).

Gene expression profile analysis. The normalized data file of GSE35452 was downloaded directly from the GEO database for further analysis. The DE genes between the responders and non-responders to neoadjuvant CRT in rectal cancer were identified by Student's t-test using the limma package of R software (version 3.4.2, https://www.r-project.org/) (12). $\mathrm{P}<0.05$ was considered to indicate a statistically significant difference. Following identification of the DE genes, bidirectional hierarchical clustering was conducted with the expression values of the DE genes using the gplots package of R software.

Functional enrichment analysis. Gene Ontology (GO) is a common method used for annotating large numbers of genes at the functional level (13). Kyoto Encyclopedia of Genes and Genomes (KEGG), a database containing known genes and their respective biochemical functionalities, can be used for systematic analysis of gene functions (14). The current study conducted KEGG pathway and GO enrichment analyses of the identified DE genes using the online version of Database for Annotation, Visualization and Integrated Discovery (DAVID) (https://david.ncifcrf.gov/). $\mathrm{P}<0.05$ was considered to indicate a statistically significant enriched term. In the present study, the functional enriched results were screened for their relevance with CRT response by searching the PubMed database (https://www.ncbi.nlm.nih.gov/pubmed).

Protein-protein interaction (PPI) network construction and analysis. A PPI network of the DE genes was constructed using the Search Tool for the Retrieval of Interacting Genes (STRING; string-db.org) database, which is a unified database of PPIs of 9,643,763 proteins from 2,031 organisms (15). The PPI network was visualized using Cytoscape 3.4.0 (http://www. cytoscape.org/). To evaluate the importance of nodes in the PPI network, three common indices, including betweenness centrality, closeness centrality and degree centrality, were investigated. The Cytoscape plug-in, CytoNCA, was used to calculate the three aforementioned indices (16). The Cytoscape plug-in, Molecular Complex Detection (MCODE), was applied to identify the significant modules of the PPI network (17). Subsequently, GO analysis and KEGG pathway analysis was performed using DAVID for the hub nodes and DE associated with the selected modules $(18) . \mathrm{P}<0.05$ was considered to indicate a statistically significant difference.

\section{Results}

Detection of DE genes between CRT responders and non-responders. Based on the aforementioned cut-off criteria $(\mathrm{P}<0.05)$, a total of 445 upregulated $\mathrm{DE}$ genes and 465 downregulated DE genes were identified in tumor samples of CRT responders and non-responders. Subsequently, hierarchical clustering analysis was performed with the 50 most upregulated and 50 most downregulated DE genes. As illustrated in Fig. 1, the DE genes could be used to clearly distinguish responders from non-responders to preoperative CRT.

Functional annotation of the DE genes. To analyze the altered biological function of the DE genes, GO enrichment analysis was performed using the DAVID online tool. The enriched GO terms were divided into biological process (BP), molecular function (MF) and cell component (CC) ontologies; the five most significantly enriched terms for each ontology are illustrated in Fig. 2. GO analysis demonstrated that upregulated DE genes in CRT responders were significantly enriched in several BP ontologies, including DNA recombination, telomere maintenance via recombination and base-excision repair. The downregulated DE genes in CRT responders were largely involved in regulatory processes, including regulation of the cAMP biosynthetic process, regulation of the extrinsic apoptotic signaling pathway and regulation of cell proliferation. For MF ontology, the upregulated DE genes in CRT responders were mainly involved in DNA binding and protein binding, and the downregulated DE genes were associated with 

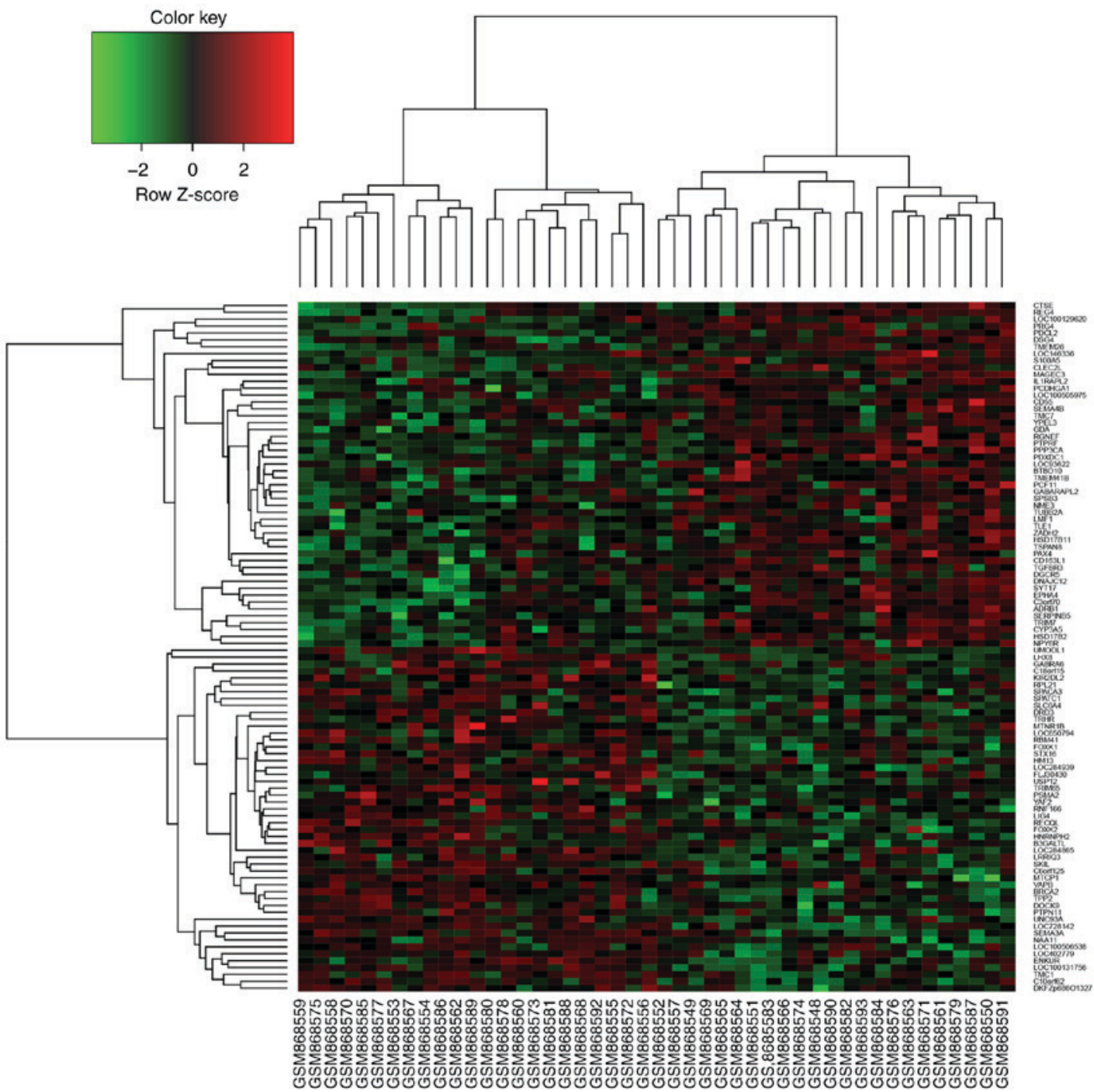

Figure 1. Heat map of the 100 most significantly differentially expressed genes (50 upregulated genes and 50 downregulated genes). Each row represents a single gene; each column represents a tissue sample. Green represents upregulated genes; red represents downregulated genes.

calcium ion binding and polysaccharide binding. In addition, GO terms in the CC ontology revealed that the upregulated DE genes in CRT responders were significantly enriched in the nucleoplasm, cytoplasm and nucleus, and downregulated DE genes in CRT responders were significantly enriched in the cytoskeleton, cytoplasmic vesicle and secretory granule.

Pathway enrichment of the DE genes. To further investigate the function of the DE genes, KEGG pathway enrichment analysis was performed using DAVID. The upregulated DE genes in CRT responders were enriched in excision repair and homologous recombination pathways, while downregulated DE genes in CRT responders were enriched in the Ras signaling pathway, the forkhead box O (FoxO) signaling pathway, focal adhesion and the Wnt signaling pathway (Table I).

PPI network construction and identification of key nodes. A PPI network of the DE genes was constructed using the STRING database. The PPI network contained 513 nodes with $910 \mathrm{DE}$ genes $(\mathrm{P}<0.001)$. The degree distributions of the network nodes are illustrated in Fig. 3. Degree centrality, betweenness centrality and closeness centrality were evaluated in the constructed network to evaluate the relationship between nodes and identify the hub genes within the network. The 10 genes with the highest degree centrality, betweenness centrality and closeness centrality are listed in Tables II-IV. Seven genes (TOP2B, MAPK8, DLG4, GCG, NGF, INSR and ARRB2) were identified as hub genes as they were identified by all three of the aforementioned methods. The sub-network was reconstructed with the selected hub nodes and their first neighbor genes, presented in Fig. 4A. The biological function of the selected hub genes were assessed and it was revealed that these genes serve a role in the Ras signaling pathway (MAPK8, INSR and NGF), the mitogen-activated protein kinase (MAPK) signaling pathway (ARRB2, MAPK8 and NGF) and the FoxO signaling pathway (MAPK8 and INSR) (Fig. 4B). Furthermore, the hub genes were identified to be involved in regulatory processes (Fig. 4C), including positive regulation of protein kinase $\mathrm{B}$ signaling (ARRB2 and INSR), positive regulation of the extracellular signal-regulated kinase (ERK) 1 and ERK2 cascades (GCG and ARRB2), positive regulation of gene expression (MAPK8 and NGF) and negative regulation of apoptotic processes (GCG and MAPK8). 


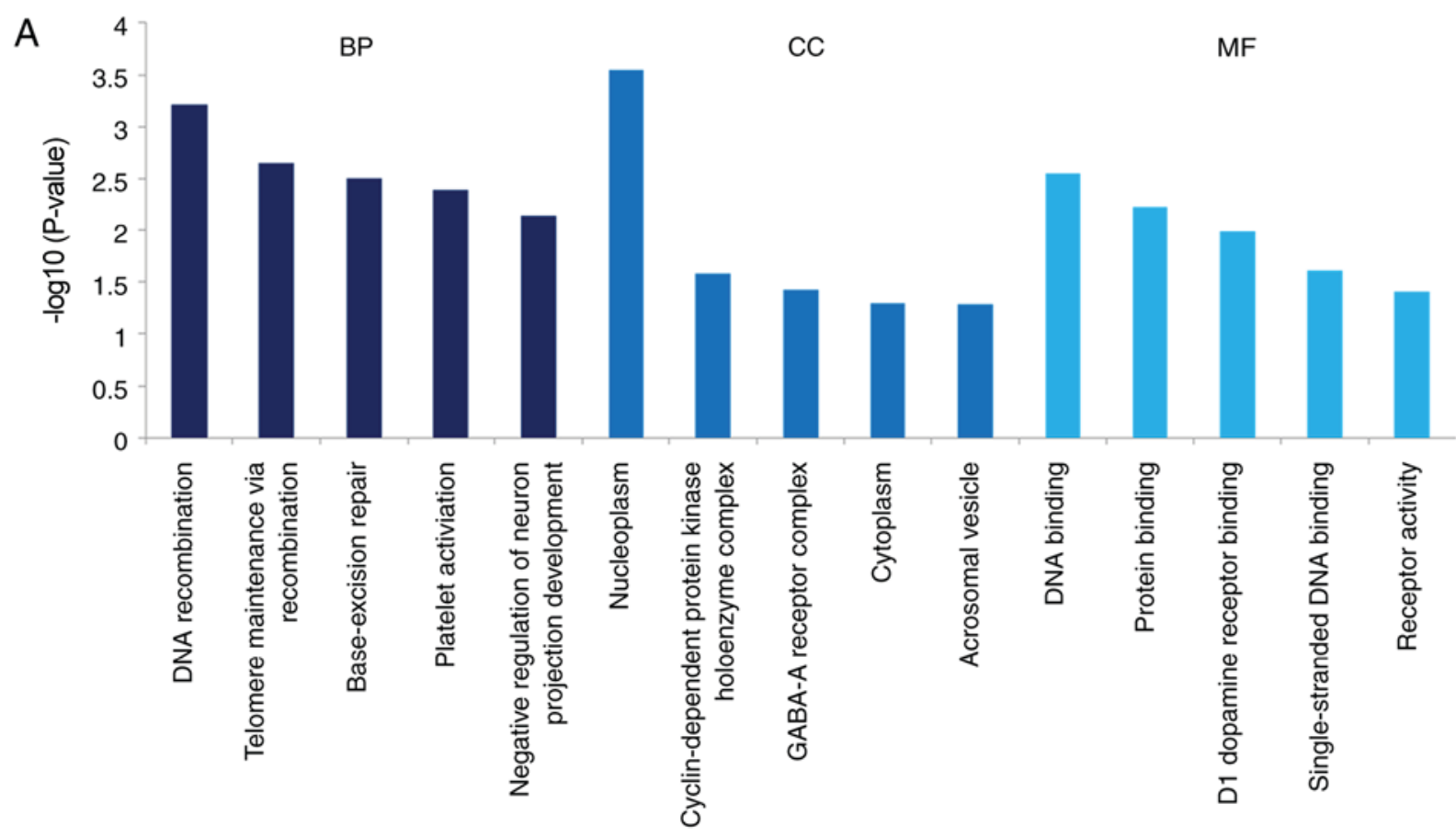

B

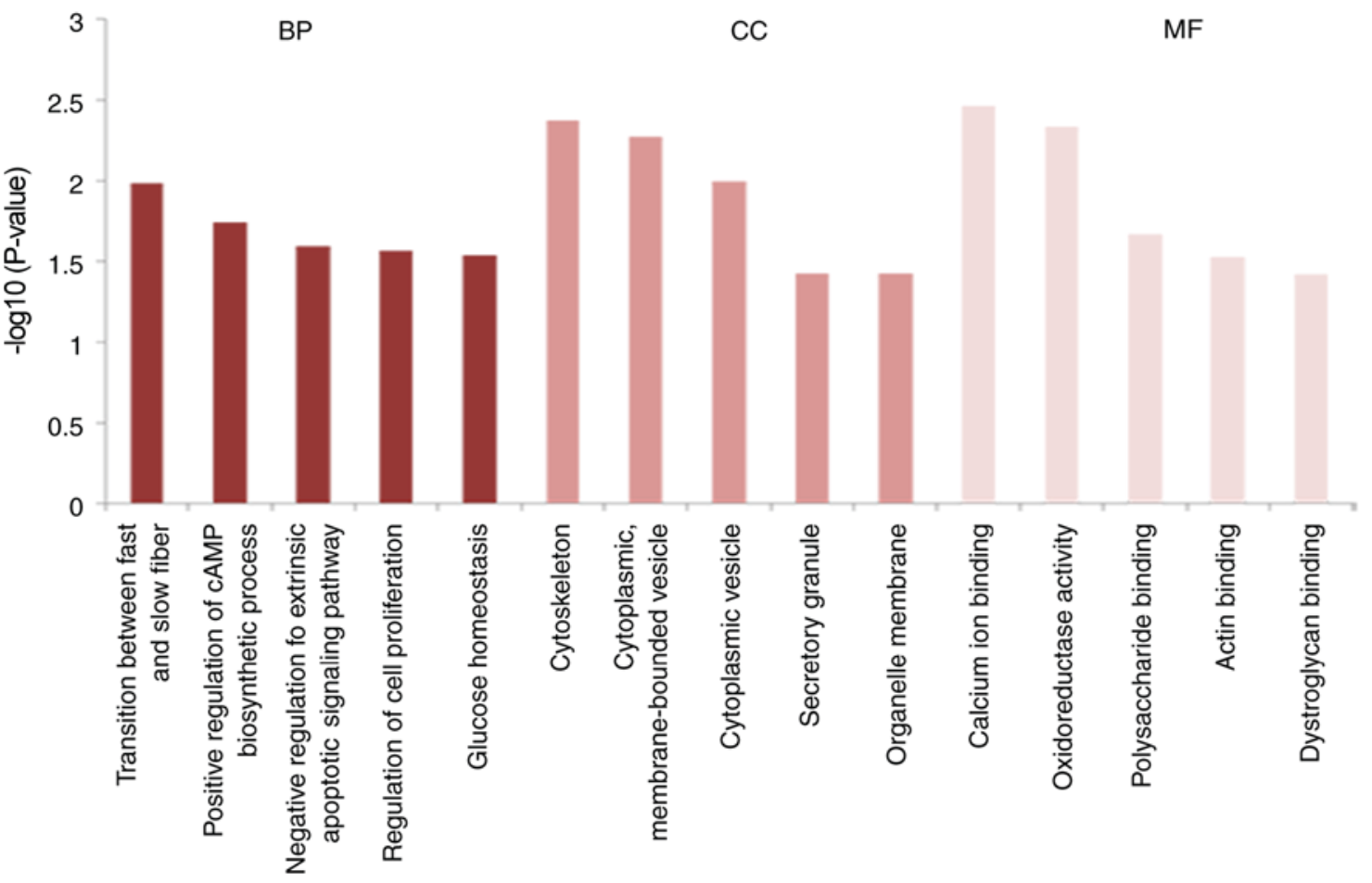

Figure 2. GO analysis of the DE genes. (A) Top five GO items for DE genes upregulated in CRT responders. (B) Top five GO items for DE genes downregulated in CRT responders. GO, Gene Ontology; BP, biological process; CC, cell component; MF, molecular function; DE, differentially expressed.

Module analysis of PPI network. The most significant modules in the PPI network were identified using MCODE in Cytoscape software and presented in Fig. 4D. According to KEGG pathway enrichment analysis, the DE genes in the significant modules were predominantly involved in several CRT response associated pathways including the chemokine signaling pathway (CCR6, ADCY5, CXCR3, CXCL11 and CCL27), cytokine-cytokine receptor interaction (CCR6, CXCR3, CXCL11 and CCL27) and the cAMP signaling pathway (HTR1B, ADCY5 and GABBR1) (Fig. 4E).
Identification of CRT response-associated functions and pathways. Based on the aforementioned results, the enriched functional categories and pathways were screened for their associations with CRT response by manually searching citations in PubMed (https://www.ncbi.nlm.nih.gov/pubmed; Table V).

For the upregulated DE genes, the most highly enriched GO terms at the BP level were associated with DNA recombination, telomere maintenance via recombination and base-excision repair. For downregulated DE genes, the more 
Table I. Kyoto Encyclopedia of Genes and Genomes pathway enrichment analysis of differentially expressed genes in chemoradiotherapy responders and non-responders.

A, Upregulated genes

\begin{tabular}{lrc}
\hline Pathway & Genes & P-value \\
\hline Neuroactive ligand-receptor interaction & 12 & $7.48 \times 10^{-3}$ \\
Base excision repair & 4 & $1.82 \times 10^{-3}$ \\
Morphine addiction & 6 & $1.94 \times 10^{-2}$ \\
GABAergic synapse & 5 & $5.70 \times 10^{-2}$ \\
mRNA surveillance pathway & 5 & $6.96 \times 10^{-2}$ \\
Measles & 6 & $7.68 \times 10^{-2}$ \\
Homologous recombination & 3 & $8.71 \times 10^{-2}$ \\
\hline
\end{tabular}

B, Downregulated genes

\begin{tabular}{lrc}
\hline Pathway & Genes & P-value \\
\hline Linoleic acid metabolism & 7 & $4.39 \times 10^{-5}$ \\
Ras signaling pathway & 12 & $3.23 \times 10^{-3}$ \\
Steroid hormone biosynthesis & 6 & $3.42 \times 10^{-3}$ \\
Oxytocin signaling pathway & 8 & $1.98 \times 10^{-3}$ \\
Renin secretion & 5 & $3.03 \times 10^{-2}$ \\
FoxO signaling pathway & 7 & $3.11 \times 10^{-2}$ \\
Arrhythmogenic right ventricular & 5 & $3.49 \times 10^{-2}$ \\
cardiomyopathy & & \\
Hypertrophic cardiomyopathy & 5 & $5.51 \times 10^{-2}$ \\
Vascular smooth muscle contraction & 6 & $6.42 \times 10^{-2}$ \\
Dilated cardiomyopathy & 5 & $6.83 \times 10^{-2}$ \\
Regulation of lipolysis in adipocytes & 4 & $7.55 \times 10^{-2}$ \\
Maturity onset diabetes of the young & 3 & $7.84 \times 10^{-2}$ \\
Fatty acid elongation & 3 & $7.84 \times 10^{-2}$ \\
Focal adhesion & 8 & $7.98 \times 10^{-2}$ \\
Alpha-Linolenic acid metabolism & 3 & $8.34 \times 10^{-2}$ \\
Butanoate metabolism & 3 & $8.92 \times 10^{-2}$ \\
Osteoclast differentiation & 6 & $9.26 \times 10^{-2}$ \\
Glycerophospholipid metabolism & 5 & $9.62 \times 10^{-2}$ \\
Wnt signaling pathway & 6 & $9.94 \times 10^{-2}$ \\
& &
\end{tabular}

FoxO, forkhead box $\mathrm{O}$.

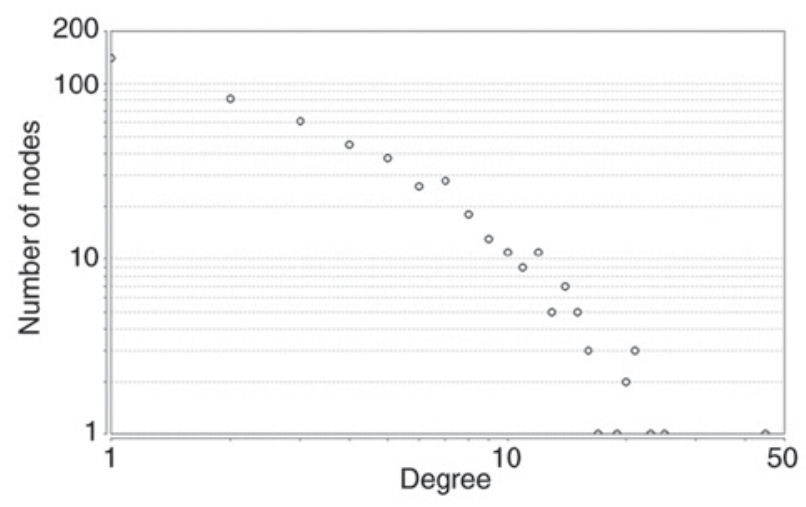

Figure 3. Degree distributions of network nodes.
Table II. Top 10 genes identified by betweenness centrality in the protein-protein interaction network.

\begin{tabular}{lc}
\hline Gene name & Betweenness centrality \\
\hline TOP2B & 50299.086 \\
MAPK8 & 27945.004 \\
DLG4 & 19934.455 \\
PPP3CA & 16902.625 \\
INSR & 15489.692 \\
GCG & 14696.811 \\
ARRB2 & 14580.526 \\
NGF & 12627.297 \\
POLR1A & 12130.832 \\
UBA2 & 11705.651 \\
\hline
\end{tabular}

Table III. Top 10 genes identified by closeness centrality in the protein-protein interaction network.

\begin{tabular}{lc}
\hline Gene name & Closeness centrality \\
\hline TOP2B & 0.020087885 \\
MAPK8 & 0.020086309 \\
INSR & 0.020060338 \\
DLG4 & 0.020056408 \\
NGF & 0.020007033 \\
GCG & 0.019985167 \\
ARRB2 & 0.019976590 \\
PCSK1 & 0.019975030 \\
RPA1 & 0.019973474 \\
HIST2H4A & 0.019967241
\end{tabular}

Table IV. Top 10 genes identified by degree centrality in the protein-protein interaction network.

\begin{tabular}{lc} 
Gene name & Degree centrality \\
\hline TOP2B & 45 \\
MAPK8 & 25 \\
DLG4 & 23 \\
ADCY5 & 21 \\
GCG & 21 \\
NGF & 21 \\
PPP3CA & 20 \\
INSR & 20 \\
ARRB2 & 19 \\
NEDD4L & 17
\end{tabular}

highly enriched GO terms at the BP level were related to regulatory processes, including cAMP biosynthetic process, extrinsic apoptotic signaling pathway and cell proliferation. Notably, these enriched terms have been demonstrated to be closely related to radiation response $(19,20)$. The enriched 


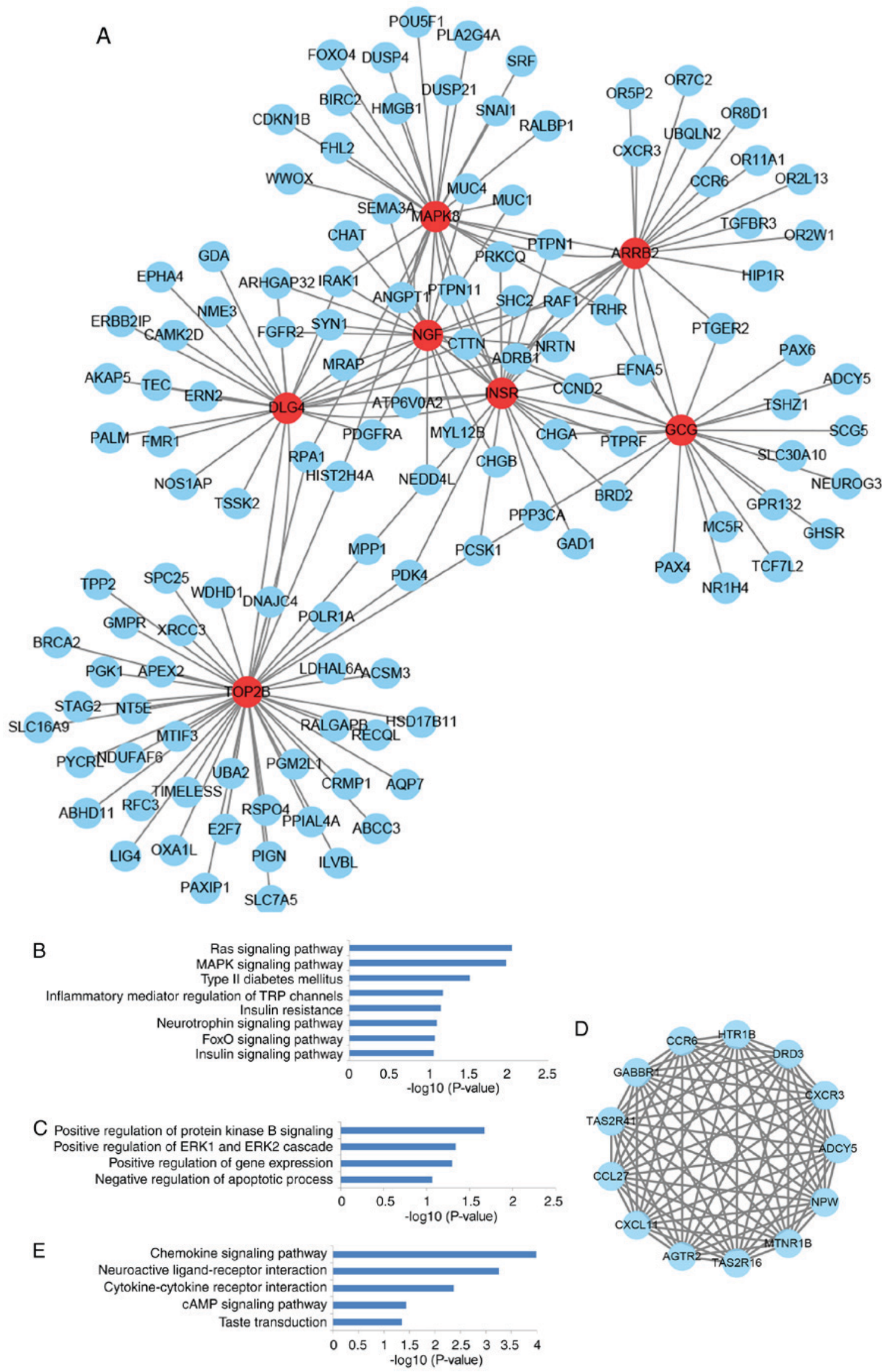

Figure 4. Analysis of the selected hub nodes and modules. (A) The sub-network reconstructed with the selected hub nodes and their first neighbor genes. (B) KEGG pathways enriched by all hub nodes. (C) Biological process ontology terms enriched by all hub nodes. (D) The most significant modules identified from the protein-protein interaction network. (E) KEGG pathways enriched by all nodes associated with the identified module with the highest significance. KEGG, Kyoto Encyclopedia of Genes and Genomes; MAPK, mitogen-activated protein kinase; TRP, transient receptor potential; FoxO, forkhead box O; ERK, extracellular signal-regulated kinase. 
Table V. Pathways associated with the chemoradiotherapy response.

\begin{tabular}{llr}
\hline Pathway & \multicolumn{1}{c}{ Enriched genes } & Representative PubMed refe \\
\hline Base excision repair & Upregulated DE genes & 21420246,20832016 \\
Homologous recombination & Upregulated DE genes & 23746696,25017126 \\
Ras signaling pathway & Downregulated DE genes and hub genes & 20619549,15549191 \\
FoxO signaling pathway & Downregulated DE genes & 17183370,20734804 \\
Focal adhesion & Downregulated DE genes and hub genes & 14703945,24930861 \\
Wnt signaling pathway & Downregulated DE genes & 27363012,26072169 \\
MAPK signaling pathway & Hub genes & 12947395,26926139 \\
Chemokine signaling pathway & Module genes & 21292447,28957436 \\
Cytokine-cytokine receptor interaction & Module genes & 28763256,27745982 \\
cAMP signaling pathway & Module genes & 24568192,26989332
\end{tabular}

FoxO, forkhead box O; MAPK, mitogen-activated protein kinase; DE, differentially expressed.

GO terms in MF mainly included the function of binding, including DNA binding, protein binding (upregulated DE genes), calcium ion binding and polysaccharide binding (downregulated DE genes). The binding of important molecules, including proteins and DNA may highly influence the ionizing radiation (21). Most GO CC items converged on the hallmarks of a cell including the nucleoplasm, cytoplasm and nucleus for the upregulated DE genes and the cytoskeleton, cytoplasmic vesicle and secretory granule for downregulated DE genes. These basic structural hallmarks of a cell have a major influence on radiation sensitivity through affecting the integrity of cell structure and exerting impact on cell growth, differentiation, proliferation, apoptosis and metastasis (22).

With regard to the KEGG pathway enrichment results, the upregulated DE genes in CRT responders were mainly involved in pathways including base excision repair and homologous recombination, while downregulated DE genes in CRT responders were enriched in pathways including the Ras signaling pathway, FoxO signaling pathway, focal adhesion and Wnt signaling pathway. These pathways were also indicated to be highly associated with radiation response (23-26). The seven hub nodes (TOP2B, MAPK8, DLG4, GCG, NGF, INSR and ARRB2) were associated with the Ras signaling pathway, MAPK signaling pathway and FoxO signaling pathway. Furthermore, the hub genes were identified to participate in specific processes, including regulation of protein kinase B signaling, ERK1 and ERK2 cascade, gene expression and apoptosis through the BP ontology. These enriched pathways and ontology terms were confirmed to be responsible for the radiation response according to the literature search $(27,28)$.

The DE genes involved in the selected module were mainly associated with the chemokine signaling pathway, cytokine-cytokine receptor interaction and cAMP signaling pathway. These pathways also play important roles in radiation response $(19,29,30)$.

\section{Discussion}

Increasing importance is being placed on improving personalized precision treatment methods for cancer. Developing methods for response prediction may help to distinguish responders from non-responders to preoperative CRT. In the current study, a bioinformatics approach was conducted to predict the potential crucial genes and pathways related to CRT response. A total of 445 upregulated DE genes and 465 downregulated DE genes were identified between CRT responders and non-responders. Subsequently, the functions of the DE genes were investigated using GO annotation and pathway enrichment analyses. Finally, the interactions between DE genes were explored by creating a PPI network. As a result, specific key genes and pathways were identified which may play vital roles in CRT response and could become potential biomarkers for the optimization of CRT.

At the BP level, many enriched GO terms were significantly associated with DNA recombination, telomere maintenance via recombination, base-excision repair, cAMP biosynthetic process, the extrinsic apoptotic signaling pathway and cell proliferation. DNA recombination is known to play a central role in the DNA damage and repair response, which is a vital determinant of both tumor development and tumor outcome following radiation therapy (31). Telomere maintenance via recombination is closely associated with telomerase activity, which is associated with radiosensitivity (32). The use of telomerase inhibitors during CRT may sensitize cancer cells to chemotherapy and radiotherapy (33). Furthermore, the cAMP biosynthetic process, the extrinsic apoptotic signaling pathway and cell proliferation also contribute to the radiotherapy response (19). The intrinsic apoptotic pathway is sufficient to impart radiation resistance (34), while inhibition of cell proliferation may enhance the radiosensitivity of cancer cells (35).

At the MF level, the enriched GO terms were mainly associated with binding functions, including DNA binding, protein binding, calcium ion binding and polysaccharide binding. These functions influence ionizing radiation through the binding of important molecules, including proteins and DNA (21). At the CC level, most GO terms involved crucial components of a cell, including the nucleoplasm, cytoplasm, nucleus, cytoskeleton, cytoplasmic vesicle and secretory granule. These structural components of a cell have a major influence on radiosensitivity (22).

KEGG pathway enrichment analysis identified several pathways associated with the upregulated DE genes in CRT 
responders, including base excision repair and homologous recombination. Meanwhile, the downregulated DE genes in CRT responders were associated with several pathways, including the Ras signaling pathway, FoxO signaling pathway, focal adhesion and Wnt signaling pathway. The base excision repair pathway is a vital determinant of ionizing radiation sensitivity and has previously been demonstrated to be associated with the cellular response to radiotherapy in many studies $(36,37)$. Targeting base excision repair may provide a potential strategy to optimize CRT (38). Previous studies have also suggested that homologous recombination repair serves an important role in the process of sensitizing cells to ionizing radiation (39). Targeting the homologous recombination pathway may be a potential translational approach for increasing sensitivity to ionizing radiation (40). Furthermore, Ras is a significant contributor to radiation resistance (41). Radiation sensitization could be achieved in vitro and in vivo by inhibiting the activation of Ras (23). Additionally, FoxO serves an important role in mediating apoptosis and regulating cell death and survival in response to radiation (24). Focal adhesion is involved in the regulation of cellular response to ionizing radiation and may be associated with increased radioresistance (25). Targeting focal adhesion kinase could provide a potential therapeutic approach for improving radiotherapy sensitivity (42). A recent study has identified that the Wnt/ $\beta$-catenin signaling pathway serves an important role in the development of radioresistance and the inhibition of Wnt/ $\beta$-catenin signaling may have significant radiosensitizing effects (26). The current study has revealed pathways that may contribute to the CRT response of rectal cancer and may be potential predictive biomarkers. The pathways identified provide a new insight into the role of the DE genes in radiation response that could help introduce therapeutic methods for radiosensitization of tumor cells.

Through PPI network construction, a series of hub genes were identified based on three different network analysis methods, including TOP2B, MAPK8, DLG4, GCG, NGF, INSR and ARRB2. It was revealed that these hub genes participate in several processes at the BP level, including the regulation of protein kinase B signaling, the ERK1 and ERK2 cascade, gene expression and apoptotic process. Protein kinase B serves a critical role in promoting the radiation-induced apoptotic response and mediating cell survival during oxidative stress, the regulation of which may have an effect on response to radiotherapy (27). The ERK1 and ERK2 cascade, gene expression and apoptotic process have also been demonstrated to exhibit synergistic roles in regulating and controlling the response to radiotherapy for rectal cancer $(20,28)$. Pathway enrichment analysis of the selected hub genes was also performed to further evaluate their function. Several significant pathways were identified, including the Ras signaling pathway, MAPK signaling pathway and FoxO signaling pathway. The MAPK signaling pathway is a vital pathway involved in radioresistance of tumor cells, therefore inhibiting the MAPK pathway may be beneficial for improving the response to radiotherapy for rectal cancer (43). The aforementioned results suggest the selected hub genes are closely associated with CRT response in rectal cancer and may be used to predict the CRT response or provide potential treatment targets for radiation sensitization.
Module analysis of the PPI network revealed that the most significant module was associated with the chemokine signaling pathway, cytokine-cytokine receptor interaction and cAMP signaling pathway. The chemokine signaling pathway has previously been reported to be responsible for mediating radiation sensitivity in tumor cells by serving a crucial role in the early phase of the radiation reaction (29). Cytokines, useful markers involved in cancer initiation, progression and pathogenesis, have previously been demonstrated to be closely associated with modulating personalized radiotherapy (30). The cAMP signaling pathway serves a role in the regulation of numerous cellular responses, including gene expression, growth, differentiation, proliferation, apoptosis and metastasis (19). A previous study has proposed that cAMP signaling contributes to the response to ionizing radiation by inhibiting DNA-damage induced apoptosis (19). Module analysis demonstrated that the most significant module of the PPI network was involved in CRT-associated pathways, further confirming that the identified DE genes were highly associated with the CRT response.

Previously, considerable effort has been made to predict and explain prognosis variability in response to CRT. However, no predictive molecular biomarkers for response to CRT have been sufficiently robust to reach the clinic. Tumors are heterogeneous and varying genes are expressed by different patients with the same cancer type. Therefore, CRT response prediction in rectal cancer cannot be determined by a single or limited number of molecules. Rectal cancer is a highly heterogeneous disease, characterized by many coordinated molecular changes; therefore potential biomarkers should be explored in a systematic and dynamic manner $(44,45)$. Gene expression profiling performed by microarray technology has successfully provided many promising biomarkers for response prediction in rectal cancer. However, the development of microarray technology has revealed that low reproducibility is common in many high-throughput post genomic areas, including proteomics and metabolomics (46). A network-based method can provide a systemic approach for integrating diverse information into a systematical framework and improve reproducibility (47). In the current study, seven gene signatures were identified from the PPI network of DE genes by using three different network analysis methods. This improves the robustness of the study, as an integrative approach allows a choice of the best biomarkers compared with a single analysis method. The identified genes and their associated pathways could potentially be developed as biomarkers for predicting CRT response. Notably, the identified genes and pathways provide potential therapeutic strategies for the radiosensitization of tumor cells.

The ideal method for screening rectal cancer CRT response-related genes is to perform microarray analysis using two groups of rectal cancer tissue samples from patients with the same treatment strategy, one from CRT sensitive tumor tissues and the other from insensitive tumor tissues. However, such tissue samples are difficult to obtain. In addition, the current study could not perform further exploration as detailed information regarding the primary histological type of cancer, staging and histological grading, and socio-demographic data were not available from the expression profile GSE35452 
database. In the future, biological experiments and large-scale investigations should be conducted to confirm the predictive power of the approach taken in the current study and to provide further understanding regarding the mechanisms underlying therapeutic outcome differences. Additionally, edge-variation in the network should be considered to further improve therapeutic response prediction.

In conclusion, the current study provided an integrative bioinformatics analysis of a gene expression profile to identify key genes and their involved pathways associated with CRT response. The current study also provided insights into the mechanisms underlying the therapeutic outcome difference, providing valuable information for both basic research and clinical optimization of CRT. However, further biological experiments are required to confirm and endorse the results presented in the current study.

\section{Acknowledgements}

Not applicable.

\section{Funding}

The present study was funded by Jiangsu Medical Innovation Team (grant no. CXDT-37), Suzhou Clinical Medical Center Construction Project (grant no. SZZXJ201503), Suzhou Science and Technology Development Program (grant no. SZS201509) and the Second Affiliated Hospital of Soochow University Preponderant Clinic Discipline Group Project funding (grant no. XKQ2015005).

\section{Availability of data and materials}

All data generated or analyzed during this study are included in this published article.

\section{Authors' contributions}

QP, KL and YS conceived the idea for the study and wrote the manuscript. QP, KL, YS, PZ and SF collected the data and analyzed the data. LZ, YTS and YZ conceived the idea for the study and revised the manuscript.

\section{Ethics approval and consent to participate}

Not applicable.

\section{Patient consent for publication}

Not applicable.

\section{Competing interests}

The authors declare that they have no competing interests.

\section{References}

1. Siegel RL, Miller KD, Fedewa SA, Ahnen DJ, Meester RGS, Barzi A and Jemal A: Colorectal cancer statistics, 2017. CA Cancer J Clin 67: 177-193, 2017.
2. Erlandsson J, Holm T, Pettersson D, Berglund Å, Cedermark B, Radu C, Johansson H, Machado M, Hjern F, Hallböök O, et al: Optimal fractionation of preoperative radiotherapy and timing to surgery for rectal cancer (Stockholm III): A multicentre, randomised, non-blinded, phase 3, non-inferiority trial. Lancet Oncol 18: 336-346, 2017.

3. Kim KH, Shin SJ, Cho MS, Ahn JB, Jung M, Kim TI, Park YS, Kim H, Kim NK and Koom WS: A phase II study of preoperative mFOLFOX6 with short-course radiotherapy in patients with locally advanced rectal cancer and liver-only metastasis. Radiother Oncol 118: 369-374, 2016.

4. Huh JW, Kim HR and Kim YJ: Clinical prediction of pathological complete response after preoperative chemoradiotherapy for rectal cancer. Dis Colon Rectum 56: 698-703, 2013.

5. Burbach JP, den Harder AM, Intven $M$, van Vulpen $M$, Verkooijen HM and Reerink O: Impact of radiotherapy boost on pathological complete response in patients with locally advanced rectal cancer: A systematic review and meta-analysis. Radiother Oncol 113: 1-9, 2014

6. Akiyoshi T, Kobunai T and Watanabe T: Predicting the response to preoperative radiation or chemoradiation by a microarray analysis of the gene expression profiles in rectal cancer. Surg Today 42: 713-719, 2012.

7. Kamensek U, Sersa G and Cemazar M: Evaluation of p21 promoter for interleukin 12 radiation induced transcriptional targeting in a mouse tumor model. Mol Cancer 12: 136, 2013.

8. Botling J, Edlund K, Lohr M, Hellwig B, Holmberg L, Lambe M, Berglund A, Ekman S, Bergqvist M, Pontén F, et al: Biomarker discovery in non-small cell lung cancer: Integrating gene expression profiling, meta-analysis, and tissue microarray validation. Clin Cancer Res 19: 194-204, 2013.

9. Zhang M, Yao C, Guo Z, Zou J, Zhang L, Xiao H, Wang D, Yang D, Gong X, Zhu J, et al: Apparently low reproducibility of true differential expression discoveries in microarray studies. Bioinformatics 24: 2057-2063, 2008.

10. Watanabe T, Kobunai T, Akiyoshi T, Matsuda K, Ishihara S and Nozawa K: Prediction of response to preoperative chemoradiotherapy in rectal cancer by using reverse transcriptase polymerase chain reaction analysis of four genes. Dis Colon Rectum 57: 23-31, 2014.

11. Trakarnsanga A, Gönen M, Shia J, Nash GM, Temple LK, Guillem JG, Paty PB, Goodman KA, Wu A, Gollub M, et al: Comparison of tumor regression grade systems for locally advanced rectal cancer after multimodality treatment. J Natl Cancer Inst 106: dju248, 2014.

12. Ritchie ME, Phipson B, Wu D, Hu Y, Law CW, Shi W and Smyth GK: limma powers differential expression analyses for RNA-sequencing and microarray studies. Nucleic Acids Res 43: e47, 2015 .

13. Gene Ontology Consortium: The Gene Ontology (GO) project in 2006. Nucleic Acids Res 34 (Database Issue): D322-D326, 2006.

14. Kanehisa M and Goto S: KEGG: Kyoto encyclopedia of genes and genomes. Nucleic Acids Res 28: 27-30, 2000.

15. Szklarczyk D, Franceschini A, Wyder S, Forslund K, Heller D, Huerta-Cepas J, Simonovic M, Roth A, Santos A, Tsafou KP, et al: STRING v10: Protein-protein interaction networks, integrated over the tree of life. Nucleic Acids Res 43 (Database Issue): D447-D452, 2015.

16. Tang Y, Li M, Wang J, Pan Y and Wu FX: CytoNCA: A cytoscape plugin for centrality analysis and evaluation of protein interaction networks. Biosystems 127: 67-72, 2015.

17. Su G, Morris JH, Demchak B and Bader GD: Biological network exploration with Cytoscape 3. Curr Protoc Bioinformatics 47: 8.13.11-24, 2014.

18. Dennis G Jr, Sherman BT, Hosack DA, Yang J, Gao W, Lane HC and Lempicki RA: DAVID: Database for annotation, visualization, and integrated discovery. Genome Biol 4: P3, 2003.

19. Cho EA, Kim EJ, Kwak SJ and Juhnn YS: cAMP signaling inhibits radiation-induced ATM phosphorylation leading to the augmentation of apoptosis in human lung cancer cells. Mol Cancer 13: 36, 2014

20. Furlong H, Mothersill C, Lyng FM and Howe O: Apoptosis is signalled early by low doses of ionising radiation in a radiation-induced bystander effect. Mutat Res 741-742: 35-43, 2013.

21. Badawi A, Hehlgans S, Pfeilschifter J, Rödel F and Eberhardt W: Silencing of the mRNA-binding protein HuR increases the sensitivity of colorectal cancer cells to ionizing radiation through upregulation of caspase-2. Cancer Lett 393: 103-112, 2017. 
22. Terry SY and Vallis KA: Relationship between chromatin structure and sensitivity to molecularly targeted auger electron radiation therapy. Int J Radiat Oncol Biol Phys 83: 1298-1305, 2012.

23. Brunner TB,Hahn SM, McKenna WG and Bernhard EJ: Radiation sensitization by inhibition of activated Ras. Strahlentherapie Onkologie 180: 731-740, 2004.

24. Luo X, Puig O, Hyun J, Bohmann D and Jasper H: Foxo and Fos regulate the decision between cell death and survival in response to UV irradiation. EMBO J 26: 380-390, 2007.

25. Beinke C, Van Beuningen D and Cordes N: Ionizing radiation modules of the expression and tyrosine phosphorylation of the focal adhesion-associated proteins focal adhesion kinase (FAK) and its substrates p130cas and paxillin in A549 human lung carcinoma cells in vitro. Int J Radiat Biol 79: 721-731, 2003.

26. Yin L, Gao Y, Zhang X, Wang J, Ding D, Zhang Y, Zhang J and Chen H: Niclosamide sensitizes triple-negative breast cancer cells to ionizing radiation in association with the inhibition of Wnt/3-catenin signaling. Oncotarget 7: 42126-42138, 2016.

27. Wang $M$, Gou $X$ and Wang L: Protein kinase B promotes radiation-induced regulatory $\mathrm{T}$ cell survival in bladder carcinoma. Scand J Immunol 76: 70-74, 2012.

28. Rahmanian N, Hosseinimehr SJ and Khalaj A: The paradox role of caspase cascade in ionizing radiation therapy. J Biomed Sci 23: 88, 2016

29. Müller K and Meineke V: Radiation-induced mast cell mediators differentially modulate chemokine release from dermal fibroblasts. J Dermatol Sci 61: 199-205, 2011.

30. Bravatà V, Minafra L, Forte GI, Cammarata FP, Russo G, Di Maggio FM, Augello G, Lio D and Gilardi MC: Cytokine profile of breast cell lines after different radiation doses. Int J Radiat Biol 93: 1217-1226, 2017.

31. Goldstein M and Kastan MB: The DNA damage response: Implications for tumor responses to radiation and chemotherapy. Annu Rev Med 66: 129-143, 2015.

32. Shim G, Ricoul M, Hempel WM, Azzam EI and Sabatier L: Crosstalk between telomere maintenance and radiation effects: A key player in the process of radiation-induced carcinogenesis Mutat Res Rev Mutat Res: S1383-5742(14)00002-7, 2014.

33. Wesbuer S, Lanvers-Kaminsky C, Duran-Seuberth I, Bölling T, Schäfer KL, Braun Y, Willich N and Greve B: Association of telomerase activity with radio- and chemosensitivity of neuroblastomas. Radiat Oncol 5: 66, 2010.

34. Crowther AJ, Ocasio JK, Fang F, Meidinger J, Wu J, Deal AM, Chang SX, Yuan H, Schmid R, Davis I and Gershon TR Radiation sensitivity in a preclinical mouse model of medulloblastoma relies on the function of the intrinsic apoptotic pathway. Cancer Res 76: 3211-3223, 2016.

35. Fang Y, DeMarco VG and Nicholl MB: Resveratrol enhances radiation sensitivity in prostate cancer by inhibiting cell proliferation and promoting cell senescence and apoptosis. Cancer Sci 103: 1090-1098, 2012.
36. Yin M, Liao Z, Liu Z, Wang LE, Gomez D, Komaki R and Wei Q: Functional polymorphisms of base excision repair genes XRCC1 and APEX1 predict risk of radiation pneumonitis in patients with non-small cell lung cancer treated with definitive radiation therapy. Int J Radiat Oncol Biol Phys 81: e67-e73, 2011.

37. Toprani SM and Das B: Radio-adaptive response of base excision repair genes and proteins in human peripheral blood mononuclear cells exposed to gamma radiation. Mutagenesis 30: 663-676, 2015.

38. Vens $\mathrm{C}$ and Begg AC: Targeting base excision repair as a sensitization strategy in radiotherapy. Semin Radiat Oncol 20: 241-249, 2010.

39. Somaiah N, Yarnold J, Lagerqvist A, Rothkamm K and Helleday T: Homologous recombination mediates cellular resistance and fraction size sensitivity to radiation therapy. Radiother Oncol 108: 155-161, 2013.

40. Lim YC, Roberts TL, Day BW, Stringer BW, Kozlov S, Fazry S, Bruce ZC, Ensbey KS, Walker DG, Boyd AW and Lavin MF: Increased sensitivity to ionizing radiation by targeting the homologous recombination pathway in glioma initiating cells. Mol Oncol 8: 1603-1615, 2014.

41. Kidd AR III, Snider JL, Martin TD, Graboski SF, Der CJ and Cox AD: Ras-related small GTPases RalA and RalB regulate cellular survival after ionizing radiation. Int J Radiat Oncol Biol Phys 78: 205-212, 2010.

42. Xu FF, Tao TQ, Wang XR, Li YZ, Song DD, Liu M and Liu XH: Cytosolic calreticulin inhibits microwave radiation-induced microvascular endothelial cell injury through the integrin-focal adhesion kinase pathway. Microcirculation 21: 717-729, 2014

43. Dent P, Yacoub A, Fisher PB, Hagan MP and Grant S: MAPK pathways in radiation responses. Oncogene 22: 5885-5896, 2003.

44. Peng Q, Zhu J, Shen P, Yao W, Lei Y, Zou L, Xu Y, Shen Y and Zhu Y: Screening candidate microRNA-mRNA regulatory pairs for predicting the response to chemoradiotherapy in rectal cancer by a bioinformatics approach. Sci Rep 7: 11312, 2017.

45. Ren X, Wang Y, Chen L, Zhang XS and Jin Q: ellipsoidFN: A tool for identifying a heterogeneous set of cancer biomarkers based on gene expressions. Nucleic Acids Res 41: e53, 2013.

46. Ransohoff DF: Lessons from controversy: Ovarian cancer screening and serum proteomics. J Natl Cancer Inst 97: 315-319, 2005.

47. Zeng T, Sun SY, Wang Y,Zhu H and Chen L: Network biomarkers reveal dysfunctional gene regulations during disease progression. FEBS J 280: 5682-5695, 2013. 